\title{
$\varphi$-Contraction in generalized probabilistic metric spaces
}

\author{
Saud M Alsulami ${ }^{1 *}$, Binayak S Choudhury ${ }^{2}$ and Pradyut Das ${ }^{2}$
}

"Correspondence: alsulami@kau.edu.sa

'Department of Mathematics, King Abdulaziz University, P.O. Box

138381, Jeddah, 21323, Saudi Arabia Full list of author information is available at the end of the article (c) 2015 Alsulami et al. This article is distributed under the terms of the Creative Commons Attribution 4.0 International License (http://creativecommons.org/licenses/by/4.0/), which permits unrestricted use, distribution, and reproduction in any medium, provided you give appropriate credit to the original author(s) and the source, provide a link to the Creative Commons license, and indicate if changes were made.

\begin{abstract}
We use the gauge function introduced by Fang to gain a fixed point result in probabilistic G-metric spaces. Our work extends some existing results. Moreover, our result is supported with an example.
\end{abstract}

MSC: $47 \mathrm{H} 10 ; 54 \mathrm{H} 25$

Keywords: generalized PM-space; Hadžić type t-norm; $\varphi$-function; Cauchy sequence; fixed point

\section{Introduction}

Probabilistic fixed point theory originated in the work of Sehgal and Bharucha-Reid [1] where they introduced a contraction mapping principle in probabilistic metric spaces. After that this line of research has developed through the works of different authors over the years. A comprehensive description of this development is given in the book of Hadžić and Pap [2]. Some more recent references are noted in [2-9].

Metric spaces are generalized in different ways creating spaces like fuzzy metric spaces, 2-metric spaces, etc. Generalized metric space is one of such spaces in which to every triple of points a nonnegative real number is attached. Such spaces were introduced by Mustafa and Sims [10]. Fixed point theory and related topics have been developed through a good number of works in recent times, some instances of which are in [11-14].

In the same vein probabilistic generalized metric spaces have been introduced by Zhou et al. [15] wherein they also proved certain primary fixed point results in these spaces. An interesting class of problems in probabilistic fixed point theory was addressed in recent times by use of gauge functions. These are control functions which have been used to extend the Sehgal contraction in probabilistic metric spaces. Some examples of these applications are in $[3,4,7,9,16,17]$. One of such gauge functions was introduced by Fang [16]. Here we use the gauge function used by Fang [16] to obtain a fixed point result in probabilistic G-metric spaces. Our result is supported with an example.

It is perceived that the study of fixed points for contractions defined by using control functions is an important category of problems in fixed point theory. One of the causes for this interest is the particularities involved in the proofs. With this motivation we work out the results in this paper.

( 


\section{Mathematical preliminaries}

In this section we discuss certain definitions and lemmas which will be necessary for establishing the results of the next section.

Definition 2.1 [18] A mapping $F: R \rightarrow R^{+}$is called a distribution function if it is nondecreasing and left continuous with $\inf _{t \in R} F(t)=0$ and $\sup _{t \in R} F(t)=1$, where $R$ is the set of real numbers and $R^{+}$denotes the set of all nonnegative real numbers.

Definition 2.2 $[2,18]$ A binary operation $\Delta:[0,1]^{2} \rightarrow[0,1]$ is called a continuous $t$-norm if the following properties are satisfied:

(i) $\Delta$ is associative and commutative,

(ii) $\Delta(a, 1)=a$ for all $a \in[0,1]$,

(iii) $\Delta(a, b) \leq \Delta(c, d)$ whenever $a \leq c$ and $b \leq d$ for all $a, b, c, d \in[0,1]$,

(iv) $\Delta$ is continuous.

Generic examples of continuous $t$-norm are $\Delta_{M}(a, b)=\min \{a, b\}, \Delta_{P}(a, b)=a b$, etc.

Definition 2.3 [18] A Menger space is a triplet $(X, F, \Delta)$, where $X$ is a nonempty set, $F$ is a function defined on $X \times X$ to the set of distribution functions and $\Delta$ is a $t$-norm such that the following are satisfied:

(i) $F_{x, y}(0)=0$ for all $x, y \in X$,

(ii) $F_{x, y}(s)=1$ for all $s>0$ if and only if $x=y$,

(iii) $F_{x, y}(s)=F_{y, x}(s)$ for all $s>0, x, y \in X$,

(iv) $F_{x, y}(u+v) \geq \Delta\left(F_{x, z}(u), F_{z, y}(v)\right)$ for all $u, v \geq 0$ and $x, y, z \in X$.

Definition 2.4 [15] The 3-tuple $(X, G, \Delta)$ is called a probabilistic G-metric space (shortly PGM-space) if $X$ is a nonempty set, $\Delta$ is a continuous $t$-norm and $F^{*}$ is a function from $X^{3} \times(0, \infty)$ to the set of distribution functions satisfying the following conditions for each $x, y, z \in X$ and $t, s>0$ :

(i) $F_{x, y, z}^{*}(t)=1$ if and only if $x=y=z$,

(ii) $F_{x, x, y}^{*}(t) \geq F_{x, y, z}^{*}(t)$ with $y \neq z$,

(iii) $F_{x, y, z}^{*}(t)=F_{p(x, y, z)}^{*}(t)$, where $p$ is a permutation function,

(iv) $\Delta\left(F_{x, a, a}^{*}(t), F_{a, y, z}^{*}(s)\right) \leq F_{x, y, z}^{*}(t+s)$.

Example 2.5 [15] Let $\left(X, F^{*}, \Delta\right)$ be a probabilistic metric space. Define a function $F^{*}$ : $X \times X \times X \rightarrow \mathbb{R}^{+}$by

$$
F_{x, y, z}^{*}(t)=\min \left\{F_{x, y}(t), F_{y, z}(t), F_{z, x}(t)\right\} \quad \text { for all } x, y, z \in X \text { and } t>0 \text {. }
$$

Then $\left(X, F^{*}, \Delta\right)$ is a probabilistic G-metric space.

For more examples of probabilistic G-metric space refer to [15].

Definition 2.6 [15] Let $\left(X, F^{*}, \Delta\right)$ be a probabilistic G-metric space and $x_{0}$ be any point in $X$. For any $\epsilon>0$ and $\lambda$ with $0<\lambda<1$, an $(\epsilon, \lambda)$-neighborhood of $x_{0}$ is the set of all points $y$ in $X$ for which $F_{x_{0}, y, y}^{*}(\epsilon)>1-\lambda$ and $F_{y, x_{0}, x_{0}}^{*}(\epsilon)>1-\lambda$. We write

$$
N_{x_{0}}(\epsilon, \lambda)=\left\{y \in X: F_{x_{0}, y, y}^{*}(\epsilon)>1-\lambda, F_{y, x_{0}, x_{0}}^{*}(\epsilon)>1-\lambda\right\} .
$$


This means that $N_{x_{0}}(\epsilon, \lambda)$ is the set of all points $y \in X$ for which the probability of the distance from $x_{0}$ to $y$ being less than $\epsilon$ is greater than $1-\lambda$.

Lemma 2.7 [15] If $\epsilon_{1} \leq \epsilon_{2}$ and $\lambda_{1} \leq \lambda_{2}$, then $N_{x_{0}}\left(\epsilon_{1}, \lambda_{1}\right) \subseteq N_{x_{0}}\left(\epsilon_{2}, \lambda_{2}\right)$.

Theorem 2.8 [15] Let $\left(X, F^{*}, \Delta\right)$ be a probabilistic G-metric space. Then $\left(X, F^{*}, \Delta\right)$ is a Hausdorff space in the topology induced by the family $\left\{N_{x_{0}}(\epsilon, \lambda)\right\}$ of $(\epsilon, \lambda)$-neighborhoods.

Definition 2.9 [15] Let $\left(X, F^{*}, \Delta\right)$ be a probabilistic G-metric space.

(i) A sequence $\left\{x_{n}\right\} \subset X$ is said to converge to a point $x \in X$ if given $\epsilon>0, \lambda>0$ we can find a positive integer $N_{\epsilon, \lambda}$ such that for all $n>N_{\epsilon, \lambda}$,

$$
F_{x, x_{n}, x_{n}}(\epsilon) \geq 1-\lambda
$$

(ii) A sequence $\left\{x_{n}\right\}$ is said to be a Cauchy sequence in $X$ if given $\epsilon>0, \lambda>0$ there exists a positive integer $N_{\epsilon, \lambda}$ such that

$$
F_{x_{n}, x_{m}, x_{l}}(\epsilon) \geq 1-\lambda \quad \text { for all } m, n, l>N_{\epsilon, \lambda}
$$

(iii) A probabilistic G-metric space $\left(X, F^{*}, \Delta\right)$ is said to be complete if every Cauchy sequence is convergent in $X$.

Definition 2.10 [2] A $t$-norm $\Delta$ is said to be a Hadžić type (shortly H-type) $t$-norm if the family $\left\{\Delta^{m}\right\}_{m>0}$ of its iterates defined for each $t \in[0,1]$ by

$$
\Delta^{1}(t)=\Delta(t, t)
$$

and, in general, for all $m>1, \Delta^{m}(t)=\Delta\left(t, \Delta^{m-1}(t)\right)$ is equi-continuous at $t=1$, that is, given $\lambda>0$ there exists $\eta(\lambda) \in(0,1)$ such that

$$
\eta(\lambda)<t \leq 1 \quad \Rightarrow \quad \Delta^{(m)}(\eta(\lambda)) \geq 1-\lambda \quad \text { for all } m>0 .
$$

Definition 2.11 [16] Let $\Phi_{w}$ denote the class of all functions $\varphi: \mathbb{R}^{+} \rightarrow \mathbb{R}^{+}$satisfying the following condition:

for each $t>0$, there exists $r \geq t$ such that $\lim _{n \rightarrow \infty} \varphi^{n}(r)=0$.

Lemma 2.12 [16] Let $\varphi \in \Phi_{w}$, then for each $t>0$ there exists $r \geq t$ such that $\varphi(r)<t$.

\section{Main results}

Definition 3.1 Let $\left(X, F^{*}, \Delta\right)$ be a PGM-space with a continuous $t$-norm $\Delta$ of H-type. A mapping $f: X \rightarrow X$ is said to be a probabilistic $\varphi$-contraction if there exists a function $\varphi \in \Phi_{w}$ such that

$$
F_{f x, f y, f z}^{*}(\varphi(t)) \geq F_{x, y, z}^{*}(t) \quad \text { for all } x, y, z \in X \text { and } t>0 \text {. }
$$


Lemma 3.2 Let $\left\{x_{n}\right\}$ be a sequence in a Menger PGM-space $\left(X, F^{*}, \Delta\right)$, where $\Delta$ is a Hadžić type $t$-norm. If there exists a function $\varphi \in \Phi_{w}$ such that

(i) $\varphi(t)>0$ for all $t>0$,

(ii) $F_{x_{n}, x_{n+1}, x_{n+1}}^{*}(\varphi(t)) \geq F_{x_{n-1}, x_{n}, x_{n}}^{*}(t)$ for all $n \in \mathbb{N}$ and $t>0$

for all $t>0, n \geq 1$, then $\left\{x_{n}\right\}$ is a Cauchy sequence in $X$.

Proof The condition (i) implies that $\varphi^{n}(t)>0$ for all $t>0$ and $n \geq 1$, and from the condition (ii), by induction, we have

$$
F_{x_{n}, x_{n+1}, x_{n+1}}^{*}\left(\varphi^{n}(t)\right) \geq F_{x_{0}, x_{1}, x_{1}}^{*}(t) \quad \text { for all } t>0, n \geq 1 .
$$

We now prove that

$$
\lim _{n \rightarrow \infty} F_{x_{n}, x_{n+1}, x_{n+1}}^{*}(t)=1 \quad \text { for all } t>0 .
$$

Since $F_{x_{0}, x_{1}, x_{1}}^{*}(t) \rightarrow 1$ as $t \rightarrow \infty$, for any $\epsilon \in(0,1)$, there exists $t_{1}>0$ such that $F_{x_{0}, x_{1}, x_{1}}^{*}\left(t_{1}\right)>$ $1-\epsilon$. Since $\varphi \in \Phi_{w}$, there exists $t_{2} \geq t_{1}$ such that $\lim _{n \rightarrow \infty} \varphi^{n}\left(t_{2}\right)=0$. Thus, for all $t>0$, there exists $n_{0} \geq 1$ such that $\varphi^{n}\left(t_{2}\right)<t$ for all $n \geq n_{0}$. By $t>\varphi^{n}\left(t_{2}\right), t_{2} \geq t_{1}$, (3.3) and the monotonic property of distribution functions, we have

$$
F_{x_{n}, x_{n+1}, x_{n+1}}^{*}(t) \geq F_{x_{n}, x_{n+1}, x_{n+1}}^{*}\left(\varphi^{n}\left(t_{2}\right)\right) \geq F_{x_{0}, x_{1}, x_{1}}^{*}\left(t_{2}\right) \geq F_{x_{0}, x_{1}, x_{1}}^{*}\left(t_{1}\right)>1-\epsilon \quad \text { for all } n \geq n_{0} .
$$

Thus (3.4) holds.

Since $\varphi \in \Phi_{w}$, by Lemma 2.12, for any $t>0$, there exists $r \geq t$ such that $\varphi(r)<t$. Let $n \geq 1$ be given. Now we show by induction that, for any $k \geq 1$,

$$
F_{x_{n}, x_{n+k}, x_{n+k}}^{*}(t) \geq \Delta^{k}\left(F_{x_{n}, x_{n+1}, x_{n+1}}^{*}(t-\varphi(r))\right) .
$$

For $k=1$, from (3.5) we have

$$
\begin{aligned}
F_{x_{n}, x_{n+1}, x_{n+1}}^{*}(t) & \geq \Delta\left(F_{x_{n}, x_{n+1}, x_{n+1}}^{*}(t-\varphi(r)), F_{x_{n}, x_{n+1}, x_{n+1}}^{*}(t-\varphi(r))\right) \\
& =\Delta^{1}\left(F_{x_{n}, x_{n+1}, x_{n+1}}^{*}(t-\varphi(r))\right) .
\end{aligned}
$$

Thus (3.5) holds for $k=1$. Assume that (3.5) holds for some $k \geq 1$. Since $\Delta$ is monotone, from (iv) in Definition 2.4, and then by (3.2) and (3.5), it follows that

$$
\begin{aligned}
F_{x_{n}, x_{n+k+1}, x_{n+k+1}}^{*}(t) & =F_{x_{n}, x_{n+k+1}, x_{n+k+1}}^{*}(t-\varphi(r)+\varphi(r)) \\
& \geq \Delta\left(F_{x_{n}, x_{n+1}, x_{n+1}}^{*}(t-\varphi(r)), F_{x_{n+1}, x_{n+k+1}, x_{n+k+1}}^{*}(\varphi(r))\right) \\
& \geq \Delta\left(F_{x_{n}, x_{n+1}, x_{n+1}}^{*}(t-\varphi(r)), F_{x_{n}, x_{n+k}, x_{n+k}}^{*}(r)\right) \quad(\text { by }(3.1)) \\
& \geq \Delta\left(F_{x_{n}, x_{n+1}, x_{n+1}}^{*}(t-\varphi(r)), F_{x_{n}, x_{n+k}, x_{n+k}}^{*}(t)\right) \quad(\text { since } r \geq t) \\
& \geq \Delta\left(F_{x_{n}, x_{n+1}, x_{n+1}}^{*}(t-\varphi(r)), \Delta^{k}\left(F_{x_{n}, x_{n+1}, x_{n+1}}^{*}(t-\varphi(r))\right)\right) \\
& =\Delta^{k+1}\left(F_{x_{n}, x_{n+1}, x_{n+1}}^{*}(t-\varphi(r))\right),
\end{aligned}
$$

which completes the conclusion. 
Next we show that $\left\{x_{n}\right\}$ is a Cauchy sequence in $X$, that is, $\lim _{m, n, l \rightarrow \infty} F_{x_{n}, x_{m}, x_{l}}^{*}(t)=1$ for all $t>0$. Let $t>0$ and $0<\epsilon<1$. Since $\left\{\Delta^{n}(t)\right\}$ is equi-continuous at $t=1$ and $\Delta^{n}(1)=1$, then there exists $\delta>0$ such that

$$
\Delta^{n}(s)>1-\epsilon \quad \text { for all } s \in(1-\delta, 1] \text { and } n \geq 1
$$

We first prove that $\lim _{n, m \rightarrow \infty} F_{x_{n}, x_{m}, x_{m}}^{*}(t) \in(1-\delta, 1]$ for all $n \geq n_{0}$. Since $t-\varphi(r)>0$, from (3.4) there is $n_{0} \geq 1$ such that $F_{x_{n}, x_{n+1}, x_{n+1}}^{*}(t-\varphi(r))>1-\delta$ for all $n \geq n_{0}$. Hence and by (3.5) we conclude that $F_{x_{n}, x_{n+k+1}, x_{n+k+1}}^{*}(t)>1-\epsilon$ for all $k \geq 1$. Thus we proved that

$$
\lim _{n, m \rightarrow \infty} F_{x_{n}, x_{m}, x_{m}}^{*}(t)=1 \quad \text { for all } t>0
$$

By (iv) with $a=x_{m}$ in Definition 2.4, we have, for all $t>0$,

$$
F_{x_{n}, x_{m}, x_{l}}^{*}(t) \geq \Delta\left\{F_{x_{n}, x_{m}, x_{m}}^{*}\left(\frac{t}{2}\right), F_{x_{m}, x_{m}, x_{l}}^{*}\left(\frac{t}{2}\right)\right\}=\Delta\left\{F_{x_{n}, x_{m}, x_{m}}^{*}\left(\frac{t}{2}\right), F_{x_{l}, x_{m}, x_{m}}^{*}\left(\frac{t}{2}\right)\right\} .
$$

From (3.5a), it follows that

$$
\begin{array}{ll}
\lim _{n, m \rightarrow \infty} F_{x_{n}, x_{m}, x_{m}}^{*}\left(\frac{t}{2}\right)=1 & \text { for all } t>0 \\
\lim _{l, m \rightarrow \infty} F_{x_{l}, x_{m}, x_{m}}^{*}\left(\frac{t}{2}\right)=1 & \text { for all } t>0 .
\end{array}
$$

Thus, by using the continuity of $\Delta$, we have

$$
\lim _{m, n, l \rightarrow \infty} F_{x_{n}, x_{m}, x_{l}}^{*}(t) \geq \Delta\left\{\lim _{n, m \rightarrow \infty} F_{x_{n}, x_{m}, x_{m}}^{*}\left(\frac{t}{2}\right), \lim _{l, m \rightarrow \infty} F_{x_{l}, x_{m}, x_{m}}^{*}\left(\frac{t}{2}\right)\right\}=\Delta(1,1)=1
$$

Therefore, we proved that

$$
\lim _{m, n, l \rightarrow \infty} F_{x_{n}, x_{m}, x_{l}}^{*}(t)=1 \quad \text { for all } t>0
$$

This shows that $\left\{x_{n}\right\}$ is a Cauchy sequence in $X$.

Corollary 3.3 [16] Let $\left\{x_{n}\right\}$ be a sequence in a Menger PM-space $(X, F, \Delta)$, where $\Delta$ is a Hadžic type $t$-norm. If there exists a function $\varphi \in \Phi_{w}$ such that

(i) $\varphi(t)>0$ for all $t>0$,

(ii) $F_{x_{n}, x_{m}}(\varphi(t)) \geq F_{x_{n-1}, x_{m-1}}(t)$

for all $t>0, n, m \geq 1$, then $\left\{x_{n}\right\}$ is a Cauchy sequence in $X$.

Proof Define $F_{x, y, z}^{*}(t)=\min \left\{F_{x, y}(t), F_{y, z}(t), F_{x, z}(t)\right\}$ for all $x, y, z \in X$ and $t>0$. Then $\left(X, F^{*}, \Delta\right)$ is a probabilistic G-metric space.

Since $F_{x_{n}, x_{n+1}, x_{n+1}}^{*}(\varphi(t))=\min \left\{F_{x_{n}, x_{n+1}}(\varphi(t)), F_{x_{n+1}, x_{n+1}}(\varphi(t)), F_{x_{n}, x_{n+1}}(\varphi(t))\right\}$, it follows

$$
F_{x_{n}, x_{n+1}, x_{n+1}}^{*}(\varphi(t))=F_{x_{n}, x_{n+1}}(\varphi(t)) .
$$


Similarly we have

$$
F_{x_{n-1}, x_{n}, x_{n}}^{*}(t)=F_{x_{n-1}, x_{n}}(t) .
$$

By using (3.7), (3.8) and (3.6), we have, for all $t>0$,

$$
F_{x_{n}, x_{n+1}, x_{n+1}}^{*}(\varphi(t))=F_{x_{n}, x_{n+1}}(\varphi(t)) \geq F_{x_{n-1}, x_{n}}(t)=F_{x_{n-1}, x_{n}, x_{n}}^{*}(t) .
$$

Hence we conclude that (3.2) holds. By Lemma 3.2, we conclude that $\left\{x_{n}\right\}$ is a Cauchy sequence in the sense of PGM-space $\left(X, F^{*}, \Delta\right)$, that is, given $\epsilon>0, \lambda>0$ there exists a positive integer $N_{\epsilon, \lambda}$ such that

$$
F_{x_{n}, x_{m}, x_{l}}^{*}(\epsilon) \geq 1-\lambda \quad \text { for all } m, n, l>N_{\epsilon, \lambda} \text {. }
$$

By definition of $F^{*}$ and (3.9), we have, for all $t>0$,

$$
\min \left\{F_{x_{n}, x_{m}}(t), F_{x_{m}, x_{l}}(t), F_{x_{n}, x_{l}}(t)\right\} \geq 1-\lambda
$$

This shows that $\left\{x_{n}\right\}$ is a Cauchy sequence in the sense of PM-space $(X, F, \Delta)$.

Lemma 3.4 Let $\left(X, F^{*}, \Delta\right)$ be a PGM-space. If there exists a function $\varphi \in \Phi_{w}$ such that

$$
F_{x, y, y}^{*}(\varphi(t)) \geq F_{x, y, y}^{*}(t)
$$

for all $t>0$ and $x, y \in X$, then $x=y$.

Proof Since $F^{*}$ is monotonic, it is obvious that from (3.10) it follows $\varphi(t)>0$ for all $t>0$. Therefore we have $\varphi^{n}(t)>0$ for all $t>0$ and $n \geq 1$. By induction, we have from (3.10) that

$$
F_{x, y, y}^{*}\left(\varphi^{n}(t)\right) \geq F_{x, y, y}^{*}(t)
$$

for all $t>0$ and $n \geq 1$.

To prove $x=y$, it is required that $F_{x, y, y}^{*}(t)=1$ for all $t>0$. Suppose, to the contrary, that there exists some $t_{0}>0$ such that $F_{x, y, y}^{*}\left(t_{0}\right)<1$. Since $\lim _{t \rightarrow \infty} F_{x, y, y}^{*}(t)=1$, there exists $t_{1}>t_{0}$ such that

$$
F_{x, y, y}^{*}(t)>F_{x, y, y}^{*}\left(t_{0}\right)
$$

for all $t \geq t_{1}$.

Since $\varphi \in \Phi_{w}$, there exists $t_{2} \geq t_{1}$ such that $\lim _{n \rightarrow \infty} \varphi^{n}\left(t_{2}\right)=0$. Therefore, we can choose large enough $n_{0} \geq 1$ such that $\varphi^{n_{0}}\left(t_{2}\right)<t_{0}$. By the monotone property of $F^{*}$, using (3.11) and (3.12), we have

$$
F_{x, y, y}^{*}\left(t_{0}\right) \geq F_{x, y, y}^{*}\left(\varphi^{n_{0}}\left(t_{2}\right)\right) \geq F_{x, y, y}^{*}\left(t_{2}\right)>F_{x, y, y}^{*}\left(t_{0}\right)
$$

which is a contradiction. Therefore $F_{x, y, y}^{*}(t)=1$ for all $t>0$. Hence $x=y$. 
Theorem 3.5 Let $\left(X, F^{*}, \Delta\right)$ be a PGM-space with a continuous $t$-norm $\Delta$ of H-type. If the mapping $f$ is a probabilistic $\varphi$-contraction, then $f$ has a unique fixed point in $X$.

Proof Let $x_{0} \in X$ be an arbitrary point $X$ and the sequence $\left\{x_{n}\right\}$ be defined as follows: $x_{n+1}=f^{n} x_{0}$ for all $n \geq 0$. Since $f$ is a probabilistic $\varphi$-contraction, by (3.1) for all $t>0$ we have

$$
\begin{aligned}
F_{x_{n}, x_{n+1}, x_{n+1}}^{*}(\varphi(t)) & =F_{f x_{n-1}, f x_{n}, f x_{n}}^{*}(\varphi(t)) \\
& \geq F_{x_{n-1}, x_{n}, x_{n}}^{*}(t) .
\end{aligned}
$$

By Lemma 3.2 we conclude that the sequence $\left\{x_{n}\right\}$ is a Cauchy sequence in $X$. Since $X$ is complete, there exists $x \in X$ such that $\lim _{n \rightarrow \infty} x_{n}=x$, that is, for all $t>0$,

$$
\lim _{n \rightarrow \infty} F_{x, x_{n}, x_{n}}^{*}(t)=1 .
$$

Now we prove that $x$ is a fixed point of $f$.

By (iv) with $a=f x_{n}$ in Definition 2.4, we have

$$
F_{f x, x, x}^{*}(t) \geq \Delta\left(F_{f x_{x} f x_{n}, f x_{n}}^{*}(\varphi(t)), F_{f x_{n}, x, x}^{*}(t-\varphi(t))\right)=\Delta\left(F_{f x, f x_{n}, f x_{n}}^{*}(\varphi(t)), F_{x_{n+1}, x, x}^{*}(t-\varphi(t))\right) .
$$

Hence, by (3.1), we get

$$
F_{f x, x, x}^{*}(t) \geq \Delta\left(F_{x, x_{n}, x_{n}}^{*}(t), F_{x_{n+1}, x, x}^{*}(t-\varphi(t))\right) .
$$

Again by (iii) and (iv) with $a=x_{n+1}$ in Definition 2.4,

$$
F_{x_{n+1}, x, x}^{*}(t-\varphi(t))=F_{x, x, x_{n+1}}^{*}(t-\varphi(t)) \geq \Delta\left(F_{x, x_{n+1}, x_{n+1}}^{*}\left(\frac{t-\varphi(t)}{2}\right), F_{x_{n+1}, x, x_{n+1}}^{*}\left(\frac{t-\varphi(t)}{2}\right)\right) .
$$

Hence, by (iii) in Definition 2.4, we have

$$
F_{x_{n+1}, x, x}^{*}(t-\varphi(t)) \geq \Delta\left(F_{x, x_{n+1}, x_{n+1}}^{*}\left(\frac{t-\varphi(t)}{2}\right), F_{x, x_{n+1}, x_{n+1}}^{*}\left(\frac{t-\varphi(t)}{2}\right)\right) .
$$

Now from (3.14) we get

$$
F_{f x, x, x}^{*}(t) \geq \Delta\left(F_{x, x_{n}, x_{n}}^{*}(t), \Delta\left(F_{x, x_{n+1}, x_{n+1}}^{*}\left(\frac{t-\varphi(t)}{2}\right), F_{x, x_{n+1}, x_{n+1}}^{*}\left(\frac{t-\varphi(t)}{2}\right)\right)\right) .
$$

Since $\lim _{n \rightarrow \infty} x_{n}=\lim _{n \rightarrow \infty} x_{n+1}=x$ and $\Delta$ is continuous, taking $n \rightarrow \infty$ in (3.15) we get, for all $t>0$,

$$
F_{f x, x, x}^{*}(t) \geq \Delta(1, \Delta(1,1))=\Delta(1,1)=1 .
$$

Hence

$$
f x=x,
$$

that is, $x$ is a fixed point of $f$. 
Next suppose that $y \neq x$ is another fixed point of $f$. Then, for all $t>0$, we have

$$
\begin{aligned}
F_{x, y, y}^{*}(\varphi(t)) & =F_{f x, f y, f y}^{*}(\varphi(t)) \\
& \geq F_{x, y, y}^{*}(t),
\end{aligned}
$$

which implies, by Lemma 3.4, that $x=y$. Therefore, $f$ has a unique fixed point in $X$. This completes the proof of the theorem.

Example 3.6 Let $X=[0, \infty)$ and $\Delta(a, b)=\min \{a, b\}$ for all $a, b \in X$. Define a function $F^{*}: X^{3} \times[0, \infty) \rightarrow[0, \infty)$ by

$$
F_{x, y, z}^{*}(t)=\frac{t}{t+G(x, y, z)}
$$

for all $x, y, z \in X$ and $G(x, y, z)=|x-y|+|y-z|+|z-x|$. Then $\left(X, F^{*}, \Delta\right)$ is a probabilistic G-metric space (see [15]).

Let $f: X \rightarrow X$ be a mapping defined by $f x=\frac{x}{2}$ and $\varphi: R^{+} \rightarrow R^{+}$be defined by

$$
\varphi(t)= \begin{cases}\frac{t}{2} & \text { if } 0 \leq t<1 \\ -\frac{t}{3}+\frac{4}{3} & \text { if } 1 \leq t \leq \frac{3}{2} \\ t-\frac{2}{3} & \text { if } \frac{3}{2}<t<\infty\end{cases}
$$

It is easy to verify that $\varphi \in \Phi_{w}$ and $\varphi(t) \geq \frac{t}{2}$ for all $t \geq 0$.

Now we show that $f$ satisfies (3.1).

We have

$$
\begin{aligned}
F_{f x, f y, f z}^{*}(\varphi(t)) & =\frac{\varphi(t)}{\varphi(t)+[|f x-f y|+|f y-f z|+|f z-f x|]} \\
& =\frac{\varphi(t)}{\varphi(t)+\left[\left|\frac{x}{2}-\frac{y}{2}\right|+\left|\frac{y}{2}-\frac{z}{2}\right|+\left|\frac{z}{2}-\frac{x}{2}\right|\right]} \\
& \geq \frac{\frac{t}{2}}{\frac{t}{2}+\left[\left|\frac{x}{2}-\frac{y}{2}\right|+\left|\frac{y}{2}-\frac{z}{2}\right|+\left|\frac{z}{2}-\frac{x}{2}\right|\right]} \\
& \geq \frac{t}{t+[|x-y|+|y-z|+|z-x|]} \\
& =F_{x, y, z}^{*}(t) .
\end{aligned}
$$

This shows that (3.1) holds and $f$ has a fixed point in $X$. The fixed point is 0 .

\section{Competing interests}

The authors declare that they have no competing interests.

\section{Authors' contributions}

All authors contributed equally to the writing of this paper. All authors read and approved the final manuscript.

\section{Author details}

'Department of Mathematics, King Abdulaziz University, P.O. Box 138381, Jeddah, 21323, Saudi Arabia. ${ }^{2}$ Department of Mathematics, Indian Institute of Engineering Science and Technology, Shibpur, B. Garden, Howrah, West Bengal 711103, India. 


\section{Acknowledgements}

This project was funded by the Deanship of Scientific Research (DSR), King Abdulaziz University, Jeddah, under Grant No. (319/130/1434). The authors, therefore, acknowledge with thanks DSR technical and financial support. The authors thank the learned referees for suggestions, which helped to improve the paper.

Received: 22 December 2014 Accepted: 25 June 2015 Published online: 22 August 2015

\section{References}

1. Sehgal, VM, Bharucha-Reid, AT: Fixed point of contraction mappings on PM space. Math. Syst. Theory 6, 97-100 (1972)

2. Hadžić, O, Pap, E: Fixed Point Theory in Probabilistic Metric Spaces. Kluwer Academic, Dordrecht (2001)

3. Choudhury, BS, Das, KP: A coincidence point result in Menger spaces using a control function. Chaos Solitons Fractals 42, 3058-3063 (2009)

4. Fang, JX: Fixed point theorems of local contraction mappings on Menger spaces. Appl. Math. Mech. 12, 363-372 (1991)

5. Fang, JX: Common fixed point theorems of compatible and weak compatible maps in Menger spaces. Nonlinear Anal. 71, 1833-1843 (2009)

6. Mihet, D: Altering distances in probabilistic Menger spaces. Nonlinear Anal. 71, 2734-2738 (2009)

7. O'Regan, D, Saadati, R: Nonlinear contraction theorems in probabilistic spaces. Appl. Math. Comput. 195, 86-93 (2008)

8. Fang, JX, Gao, Y: Common fixed point theorems under strict contractive conditions in Menger spaces. Nonlinear Anal. 70, 184-193 (2009)

9. Ćirić, L: Solving the Banach fixed point principle for nonlinear contractions in probabilistic metric spaces. Nonlinear Anal. 72, 2009-2018 (2010)

10. Mustafa, Z, Sims, B: A new approach to generalized metric spaces. J. Nonlinear Convex Anal. 7, 289-297 (2006)

11. Mustafa, Z, Obiedat, H, Awawdeh, H: Some fixed point theorem for mapping on complete G-metric spaces. Fixed Point Theory Appl. 2008, Article ID 189870 (2008)

12. Mustafa, Z, Shatanawi, W, Bataineh, M: Existence of fixed point results in G-metric spaces. Fixed Point Theory Appl. 2009, Article ID 283028 (2009)

13. Mustafa, Z, Sims, B: Fixed point theorems for contractive mappings in complete G-metric spaces. Fixed Point Theory Appl. 2009, Article ID 917175 (2009)

14. Ćirić, L, Agarwal, RP, Samet, B: Mixed monotone generalized contractions in partially ordered probabilistic metric spaces. Fixed Point Theory Appl. 2011, 56 (2011)

15. Zhou, C, Wang, S, Ćirić, L, Alsulami, SM: Generalized probabilistic metric spaces and fixed point theorems. Fixed Point Theory Appl. 2014, 91 (2014)

16. Fang, JX: On $\varphi$-contractions in probabilistic and fuzzy metric spaces. Fuzzy Sets Syst. (2015). doi:10.1016/j.fss.2014.06.013

17. Jachymski, J: On probabilistic $\varphi$-contractions on Menger spaces. Nonlinear Anal. 73, 1131-1137 (2010)

18. Schweizer, B, Sklar, A: Probabilistic Metric Spaces. North-Holland, New York (1983)

\section{Submit your manuscript to a SpringerOpen ${ }^{\ominus}$ journal and benefit from:}

- Convenient online submission

Rigorous peer review

- Immediate publication on acceptance

- Open access: articles freely available online

- High visibility within the field

- Retaining the copyright to your article 